\title{
Verwendung von Leichen und Leichenteilen in der medizinischen Forschung sowie Aus-, Weiter- und Fortbildung
}

Vom Senat verabschiedet am 27. November 2008, angepasst Ende 2014.

1 Diese Empfehlungen wurden vom Senat der Schweizerischen Akademie der Medizinischen Wissenschaften (SAMW) am 27. November 2008 genehmigt; sie wurden Ende 2014 an die gesetzlichen Rahmenbedingungen angepasst.

Der Arbeitsgruppe, welche diese Empfehlungen ausgearbeitet hat, gehörten folgende Persönlichkeiten an: Prof. Volker Dittmann, Basel (Vorsitz); Prof. Jean Henri Fasel, Genf; Prof. Jean-Pierre Hornung, Lausanne; Me Charles Joye, Lausanne; Prof. Robert Maurer, Illnau; Prof. Brigitte Tag, Zürich. Die deutsche Version ist die Stammversion. 


\section{Einleitung und Problembeschreibung}

Die Verwendung von Leichen und Leichenteilen in der medizinischen Forschung sowie Aus-, Weiter- und Fortbildung ist gängige Praxis und geht auf eine teils jahrhundertealte Tradition zurück. Eine Umfrage der SAMW hat ergeben, dass in nahezu allen schweizerischen Instituten für Anatomie, Pathologie und Rechtsmedizin Forschung sowie Unterricht an Leichen erfolgt. Probleme entstehen in der Regel dann, wenn zum Beispiel im Zusammenhang mit einer rechtsmedizinischen oder klinischen Obduktion an einem Leichnam Handlungen wie Punktions-, Intubations- oder Operationsübungen vorgenommen werden, die über die routinemässige pathologisch-anatomische Diagnostik hinausgehen.

Unproblematisch erscheint demgegenüber die Verwendung von Leichen in anatomischen Instituten zu Forschungs- und Unterrichtszwecken, soweit ein auf einer angemessenen Aufklärung beruhendes schriftliches Vermächtnis des Körperspenders vorliegt.

Nicht nur in Fachkreisen sondern auch in der Öffentlichkeit haben Medienberichte für Beunruhigung und Empörung gesorgt, die dargestellt haben, dass angeblich weltweit ein schwunghafter Handel mit Leichenteilen zweifelhafter Herkunft erfolgt ${ }^{2}$. Auch in der Schweiz wurde ein Fall bekannt, bei dem aus den USA 40 tiefgefrorene Füsse für Operationsübungen importiert worden waren. In der folgenden Diskussion wurde deutlich, dass eine verbreitete Unkenntnis über bereits bestehende Regelungen herrscht ${ }^{3}$.

Aufgrund einer konkreten Anfrage zum korrekten Vorgehen insbesondere bei der an sich erwünschten und vielfach notwendigen Kooperation von Universitätsinstituten mit Partnern aus der Industrie hat sich die SAMW daher entschlossen, eine Arbeitsgruppe zu beauftragen, Empfehlungen für den Umgang mit Leichen und Leichenteilen in der medizinischen Forschung sowie Aus-, Weiter- und Fortbildung zu erarbeiten. Ziel der Empfehlungen ist nicht eine zwingende Normierung des Verhaltens; vielmehr sollen sie die Akteure in diesem Bereich in die Lage versetzen, ihr Handeln so zu gestalten, dass weder aus ethischer noch aus rechtlicher Sicht Einwände bestehen.

2 Die Zeit, 15. 2. 2007: Frische Leichenteile weltweit; Tagesspiegel, 11.10. 2006: Illegaler Handel mit Leichenteilen.

3 NZZ am Sonntag, 12. 2. 2006: Die Schweiz importierte Leichenteile; OnlineRreports.ch, 28. 2. 2006 : 40 Füsse von US-Leichen erhitzen die politischen Gemüter. 


\section{Rechtliche Rahmenbedingungen}

Der Umgang mit der Leiche ist nicht abschliessend geregelt. Es gelten internationale, europäische und nationale Normen, die sich zum Teil ergänzen, überschneiden und dennoch den Umgang mit der Leiche nur fragmentarisch regeln.

Nach dem Übereinkommen über Menschenrechte und Biomedizin (kurz: BMÜ) ${ }^{4}$ und dem Zusatzprotokoll über die Transplantation von Organen und Geweben menschlichen Ursprungs (kurz: TP) ${ }^{5}$ dürfen der menschliche Körper und Teile davon nicht zur Gewinnerzielung verwendet werden ${ }^{6}$; davon nicht betroffen ist die Abgeltung notwendiger Aufwendungen für Material und Dienstleistungen. Zudem gilt grundsätzlich ein Handelsverbot. ${ }^{7}$ Die Entnahme von Teilen des menschlichen Körpers bedarf grundsätzlich der Einwilligung. ${ }^{8}$ Zudem dürfen sie nur zu dem Zweck aufbewahrt werden, zu dem sie entnommen wurden; jede andere Verwendung setzt angemessene Informations- und Einwilligungsverfahren voraus. ${ }^{9}$ Teilregelungen zum Umgang mit der Leiche finden sich auch im EU-Recht, insbesondere der EU- Richtlinie 2004/23/EG ${ }^{10}$ sowie den hierauf bezogenen Ausführungsbestimmungen. ${ }^{11}$ Sie regeln u.a. die Rahmenbedingungen für die Transplantationsmedizin und die Forschung mit Substanzen Verstorbener.

Im nationalen Recht sind Teilbereiche zum Umgang mit der Leiche in verschiedenen Gesetzen geregelt. Die Bundesverfassung schützt die Leiche durch die Artikel zur Menschenwürde ${ }^{12}$, Schutz der Gesundheit ${ }^{13}$, Transplantationsmedizin ${ }^{14}$, Zivilrecht, Strafrecht ${ }^{15}$ und Wirtschaftsfreiheit ${ }^{16}$. Seit dem 1. Juli 2007 gilt zudem das Transplantationsgesetz. ${ }^{17}$ Es soll den missbräuchlichen Umgang der Transplantationsmedizin beim Menschen, insbesondere den Handel mit den Körpersubstanzen, verhindern und die Menschenwürde, die Persönlichkeit und die Gesundheit schützen..$^{18}$ Die wesentlichen Grundsätze der postmortalen Spende sind

4 Übereinkommen zum Schutz der Menschenrechte und der Menschenwürde im Hinblick auf die Anwendung von Biologie und Medizin. Die Schweiz hat das Übereinkommen am 7. Mai 1999 unterzeichnet.

5 Strassburg, 24. Januar 2002, ETS Nr. 186. Auch das vom Ministerkomitee am 8. November 2001 verabschiedete, am 1. Mai 2006 in Kraft getretene Zusatzprotokoll wurde von der Schweiz am 11. Juli 2002 unterzeichnet. Beide Texte wurden von der Schweiz zwar unterschrieben, aber noch nicht ratifiziert.

6 Art. 21 BMÜ.

7 Art. 21, 22 TP.

8 Vgl. Art. 16 - 18 BMÜ.

9 Art. 22 BMÜ.

10 Zur Festlegung von Qualitäts- und Sicherheitsstandards für die Spende, Beschaffung, Testung, Verarbeitung, Konservierung, Lagerung und Verteilung von menschlichen Geweben und Zellen, vgl. www.bag.admin.ch/transplantation/00694/00727/01859/index.html?lang=de.

11 EU-Richtlinie 2006/17/EG, Richtlinie 2004/23/EG sowie die Empfehlungen des Europarates zur Forschung mit biologischem Material vom 15.3.2006.

12 Art. 7 BV.

13 Art. 118 BV.

14 Art. 119a BV.

15 Art. 122, Art. 123 BV.

16 Art. 27, Art. 94 und Art. 95 BV.

17 Bundesgesetz vom 8. Oktober 2004 über die Transplantation von Organen, Geweben und Zellen (Transplantationsgesetz), SR 810.21 sowie die hierauf bezogenen Verordnungen.

18 Art. 1 Abs. 3 Transplantationsgesetz. 
auf der Grundlage der erweiterten Zustimmungslösung in Art. 8 ff. Transplantationsgesetz geregelt. ${ }^{19}$ Am 1. Januar 2014 ist das Bundesgesetz über die Forschung mit Menschen ${ }^{20}$ in Kraft getreten, welches auch Regelungen zur Forschung mit verstorbenen Personen enthält (vgl. Kap. 3.2.).

Der Umgang mit der Leiche hat weiterhin Berührungspunkte zum Datenschutzrecht des Bundes und der Kantone und zur Heilmittelgesetzgebung. Darüber hinaus schützt das Strafgesetzbuch die Leiche vor einigen unbefugten Eingriffen. Gemäss Art. 262 StGB ist u.a. strafbar, wer einen Leichnam verunehrt oder öffentlich beschimpft oder wer einen Leichnam oder Teile eines Leichnams gegen den Willen des Berechtigten wegnimmt. Zusätzlich können weitere Tatbestände relevant sein, so z.B. die ärztliche Schweigepflicht, Art. 321 StGB, das Forschungsgeheimnis, Art. 321 ${ }^{\text {bis }}$ StGB, sowie die Eigentums- und Vermögensdelikte, Art. 137, 139 StGB. Auch das Zivilrecht hält allgemeine Bestimmungen zum Umgang mit der Leiche bereit. Zu erwähnen sind der Schutz der Persönlichkeit ${ }^{21}$ oder die allgemeinen Bestimmungen des Sachenrechts. ${ }^{22}$ Die Frage, ob die Leiche eigentumsfähig oder herrenlos ist und unter welchen Voraussetzungen Gewebe, das legal entnommen wurde, für Forschungs- oder sonstige Zwecke aufbewahrt und weiterverwendet werden darf, ist bislang aber nicht hinlänglich beantwortet.

Darüber hinaus fällt der Umgang mit dem toten Körper in die Gesetzgebungskompetenz der Kantone. Das Bestattungswesen ist in vielfältigen kantonalen und kommunalen Erlassen normiert, die Gesundheitsgesetze sowie Patientinnenund Patientengesetze enthalten zudem Sonderregelungen. ${ }^{23}$ Ansonsten begrenzen kantonale Polizeigesetze den Umgang mit der Leiche, indem dadurch z.B. unmittelbar drohende oder eingetretene schwere Störungen der öffentlichen Sicherheit und Ordnung abgewehrt oder beseitigt werden können.

19 D.h. die postmortale Entnahme der Körpersubstanzen ist an die Bedingung geknüpft, dass die spendende Person vor ihrem Tod der Entnahme zugestimmt hat, Art. 8 Abs. 1. Liegt keine Willensäusserung vor, so geht das Bestimmungsrecht auf die nächsten Angehörigen über. Sie können unter Beachtung des mutmasslichen Willens der verstorbenen Person über das "ob» und "den Umfang" der Organentnahme entscheiden.

20 Bundesgesetz über die Forschung am Menschen vom 30. September 2011 (Humanforschungsgesetz), SR 810.30.

21 Z.B. Art. 27 und 28 ZGB.

22 Art. $641 \mathrm{ff}$. ZGB.

23 Eine Zusammenstellung findet sich bei Bär W, Keller-Sutter M, Leichenschau, Obduktion und Transplantation, in: Poledna MW, Kuhn T (Hrsg.), Arztrecht in der Praxis, 2. Aufl. 2007, S. 767, 775 f. 


\section{Empfehlungen}

\section{1. "Informierte Einwilligung" als Prinzip}

In der modernen biomedizinischen Ethik ist unbestritten, dass es grundsätzlich bei allen Eingriffen in die körperliche Integrität, sei es zu Lebzeiten oder nach dem Tode, zur Wahrung der Persönlichkeitsrechte jedes Menschen einer rechtswirksamen Einwilligung bedarf. Diese setzt eine angemessene Aufklärung über alle wichtigen Umstände des geplanten Eingriffs voraus (informed consent). Hierzu gehören insbesondere auch Informationen über Art, Umfang und Folgen eines Eingriffs. Hat ein Verstorbener zu Lebzeiten keine entsprechende Erklärung abgegeben, so können auch die nächsten Angehörigen nach entsprechender Aufklärung zustimmen, wobei sie den mutmasslichen Willen des Verstorbenen zu beachten haben. Im Zweifelsfall hat der Wille der verstorbenen Person Vorrang vor dem der Angehörigen. Aus dem Umstand, dass es in der Regel Aufgabe des (letzt) behandelnden Arztes (und nicht des Pathologen) ist, die notwendige Einwilligung einzuholen, können Konfliktfelder entstehen, die es anzusprechen gilt.

\subsection{Forschung}

Die Forschung zu Krankheiten des Menschen sowie zu Aufbau und Funktion des menschlichen Körpers, die an verstorbenen Personen durchgeführt wird, ist seit dem 1. Januar 2014 abschliessend im Bundesgesetz über die Forschung am Menschen (HFG) geregelt. ${ }^{24}$ Sie darf durchgeführt werden, wenn die betreffende Person vor ihrem Tod in die Verwendung ihres Körpers zu Forschungszwecken eingewilligt hat. Liegt keine dokumentierte Einwilligung oder Ablehnung der verstorbenen Person vor, so dürfen ihr Körper oder dessen Teile zu Forschungszwecken verwendet werden, wenn die nächsten Angehörigen oder eine von der verstorbenen Person zu Lebzeiten bezeichnete Vertrauensperson einwilligen. ${ }^{25}$

Weniger strenge Regelungen gelten, wenn im Rahmen einer Obduktion oder Transplantation geringfügige Mengen von Körpersubstanzen für ein Forschungsprojekt entnommen werden oder wenn der Tod einer Person länger als 70 Jahre zurückliegt. 
So darf eine geringfügige Menge von Körpersubstanzen ohne Einwilligung anonymisiert und für Forschung verwendet werden, sofern keine dokumentierte Ablehnung der verstorbenen Person vorliegt. ${ }^{26}$ Der Gesetzgeber spricht von Blut- oder Gewebeproben im Milliliter- oder Grammbereich ${ }^{27}$ und begründet diese Ausnahme damit, dass der Leichnam aufgrund des Forschungsprojekts nicht zusätzlich beschädigt wird und deshalb davon ausgegangen werden kann, dass die Persönlichkeitsrechte der Angehörigen nicht verletzt werden. ${ }^{28}$

Ebenfalls ohne Einwilligung darf Forschung durchgeführt werden, wenn der Tod der verstorbenen Person länger als 70 Jahre zurück liegt und keine Ablehnung der verstorbenen Person dokumentiert ist. Wenden sich die Angehörigen gegen die Durchführung des Forschungsprojekts, darf dieses nicht durchgeführt werden.

Die Forschungsprojekte mit verstorbenen Personen müssen vorgängig durch die zuständige Ethikkommission für Forschung bewilligt werden.

\subsection{Aus-, Weiter- und Fortbildung von medizinischem Fachpersonal}

Neben der Lehre im Rahmen des Studiums der medizinischen Fächer gehört zweifellos auch die Weiter- und Fortbildung zum genuinen durch Gesetze und Reglemente definierten Aufgabenbereich anatomischer, rechtsmedizinischer und pathologischer Institute. Auch hier gelten die für die Forschung dargestellten Bedingungen, wobei es für die zustimmungsberechtigte Person durchaus einen Unterschied machen kann, ob die Einwilligung nur für die Forschung oder auch für Unterricht und Lehre erteilt werden soll. Daraus folgt, dass eine Einwilligung für die Verwendung menschlichen biologischen Materials im Rahmen von Forschungsprojekten nicht automatisch auch die Zustimmung zur Verwendung in Lehr- und Fortbildungsveranstaltungen impliziert und umgekehrt, das heisst, die Einwilligungserklärungen müssen entsprechend präzise abgefasst sein. Lehrveranstaltungen in unmittelbarem Zusammenhang mit Obduktionen (wie z.B. klinischpathologische Demonstrationen) bedürfen keiner gesonderten Zustimmung.

Im Gegensatz zu den meisten anderen Instituten, die mit Verstorbenen arbeiten, verfügen die schweizerischen anatomischen Institute über vergleichbare schriftliche Erklärungen zur Körperspende, in denen nicht nur auf die Verwendung des Körpers in der Forschung, sondern auch auf die Ausbildung von Ärzten hingewiesen wird. Zudem werden entweder ausführliche schriftliche Begleitinformationen abgegeben oder ein persönliches Informationsgespräch angeboten, in denen alle notwendigen Details dargestellt werden. Die SAMW empfiehlt den anderen Instituten, die Leichen oder Leichenteile in der Aus-, Weiter- oder Fortbildung verwenden, analoge Vorgehensweisen anzuwenden. 


\subsection{Kurse in der Verantwortung externer Anbieter}

Zwischen der Industrie und den anatomischen, pathologischen und rechtsmedizinischen Instituten besteht an vielen Orten eine intensive Zusammenarbeit. Diese ist insbesondere im Sinne einer Innovation von Medizinprodukten wie zum Beispiel Implantaten oder der Optimierung von Operationsverfahren auch im Interesse zukünftiger Patienten notwendig und durchaus erwünscht. Aus Sicht der SAMW bestehen keine Bedenken dagegen, wenn die Institute für derartige Kurse Räume, Material und Personal zur Verfügung stellen oder Kurse an anderen geeigneten Örtlichkeiten durchgeführt werden, soweit dabei die Verantwortlichkeiten eindeutig festgelegt und die in diesen Empfehlungen dargestellten Regeln eingehalten werden.

\subsection{Sammlung und Weitergabe von Leichenteilen inklusive Import}

Grundsätzlich gelten auch hier die bereits aufgeführten Grundsätze. Demnach ist auch der Import von Leichen oder Leichenteilen möglich. Voraussetzung ist aber, dass die in der Schweiz verantwortliche Person über eine hinreichend ausführliche schriftliche Erklärung des Lieferanten verfügt, aus der hervorgeht, dass für alle gelieferten Leichen oder Leichenteile rechtsgültige Erklärungen der jeweiligen Spender oder Zustimmungsberechtigten vorliegen, die den in der Schweiz geltenden Bestimmungen entsprechen und aus denen insbesondere hervorgeht, dass die Spender auch mit einem Export ins Ausland einverstanden waren. 


\section{Rechtliche Rahmenbedingungen zur Verwendung von Leichen und Leichenteilen in der medizinischen Forschung sowie Aus-, Weiter- und Fortbildung}

Prof. Dr. iur. utr. Brigitte Tag, Universität Zürich

\section{Einführung}

Der Umgang mit der Leiche ist nicht in einem einheitlichen Gesetz abschliessend geregelt. Vielmehr finden sich sowohl auf internationaler, europäischer wie nationaler Ebene Normen, die sich zum Teil ergänzen und überschneiden, dennoch aber nur fragmentarisch den Umgang mit dem toten menschlichen Körper regeln.

\section{Europäische Regelungen}

Zentrale Aussagen zum Umgang mit Substanzen menschlicher Herkunft liefern das Übereinkommen über Menschenrechte und Biomedizin oder Bioethikkonvention $(B M \ddot{U})^{1}$ und das Zusatzprotokoll des Europarates über die Transplantation von Organen und Geweben menschlichen Ursprungs, kurz Transplantationsprotokoll (TP). ${ }^{2}$ Beide Texte wurden von der Schweiz unterschrieben und ratifiziert. ${ }^{3}$ Art. 21 BMÜ bestimmt, dass der menschliche Körper und Teile davon als solche nicht zur Erzielung eines finanziellen Gewinns verwendet werden dürfen. Ergänzend regeln Art. 21, 22 TP das Handelsverbot und seine Grenzen. Art. 22 BMÜ betrifft den Schutz der Weiterverwendung von menschlichen Körpern und Teilen davon. Die Norm regelt explizit, dass, wenn bei einer Intervention ein Teil des menschlichen Körpers entnommen wird, er nur zu dem Zweck aufbewahrt werden darf, zu dem er entnommen worden ist; jede andere Verwendung setzt angemessene Informations- und Einwilligungsverfahren voraus. Art. 16-18 regeln die Rahmenbedingungen der postmortalen Entnahme von Organen und Geweben, namentlich die Feststellung des Todes, die nach der Rechtsordnung erforderliche Einwilligung in die Organ- und Gewebeentnahme sowie die Achtung des menschlichen Körpers.

1 Übereinkommen zum Schutz der Menschenrechte und der Menschenwürde im Hinblick auf die Anwendung von Biologie und Medizin. Die Schweiz hat das Übereinkommen am 7. Mai 1999 unterzeichnet.

2 Strassburg, 24. Januar 2002, ETS Nr. 186. Auch das vom Ministerkomitee am 8. November 2001 verabschiedete, am 1. Mai 2006 in Kraft getretene Zusatzprotokoll wurde von der Schweiz am 11. Juli 2002 unterzeichnet.

3 Vgl. Bundesbeschluss über die Genehmigung des Übereinkommens über Menschenrechte und Biomedizin vom 20. März 2008, SR 810.21. Zu den Erklärungen und Vorbehalten der Schweiz vgl. http://conventions.coe.int/Treaty/Commun/ListeDeclarations.asp?NT=186\&CM=8\&DF=23/08/2010\&CL= GER\&VL=1 
Darüber hinaus finden sich Teilregelungen zum gesollten Umgang mit der Leiche im EU-Recht. Im Vordergrund steht die EU-Richtlinie 2004/23/EG zur Festlegung von Qualitäts- und Sicherheitsstandards für die Spende, Beschaffung, Testung, Verarbeitung, Konservierung, Lagerung und Verteilung von menschlichen Geweben und Zellen. Diese Richtlinie gilt für Gewebe und Zellen zur Verwendung beim Menschen, einschliesslich menschlicher Gewebe und Zellen, die für die Zubereitung von kosmetischen Mitteln verwendet werden. Sie gilt nicht für die forschungsbedingte Nutzung menschlicher Gewebe und Zellen, es sei denn, diese Substanzen menschlicher Herkunft sollen in klinischen Versuchen im oder am menschlichen Körper eingesetzt werden. Die EU-Richtlinie 2006/17/EG regelt die Durchführung der Richtlinie 2004/23/EG hinsichtlich technischer Vorschriften für die Spende, Beschaffung und Testung von menschlichen Geweben und Zellen. Die Richtlinie 2006/86/EG der Kommission vom 24.10.2006 dient der Umsetzung der Richtlinie 2004/23/EG hinsichtlich der Anforderungen an die Rückverfolgbarkeit, der Meldung schwerwiegender Zwischenfälle und unerwünschter Reaktionen sowie bestimmter technischer Anforderungen an die Kodierung, Verarbeitung, Konservierung, Lagerung und Verteilung von menschlichen Geweben und Zellen. ${ }^{4}$ Diese Richtlinien unterbreiten u.a. Rahmenbedingungen für die Transplantationsmedizin und die Forschung mit Substanzen Verstorbener. Sie spiegeln sich daher im Transplantationsgesetz wie dem Entwurf eines künftigen Humanforschungsgesetzes wider. Die am 15.3.2006 vom Ministerkomitee des Europarates verabschiedeten Empfehlungen des Europarates zur Forschung mit biologischem Material unterstreichen in Art. 7 das bereits in Art. 21 BMÜ festgelegte Verbot finanziellen Gewinns bezogen auf biologisches Material. Art. 13 bestimmt, dass die postmortale Entnahme von Körpermaterial zu Forschungszwecken nicht ohne entsprechende Einwilligung bzw. Bewilligung geschehen und bei Widerspruch des Verstorbenen zu Lebzeiten nicht erfolgen darf. Darüber hinaus werden Grundsätze zur Aufbewahrung von Substanzen menschlicher Herkunft in Biobanken sowie zur Forschung mit diesen Substanzen aufgestellt, die grundsätzlich auch für postmortal gewonnene Substanzen und Daten Anwendung finden. 


\section{Nationales Recht}

Im nationalen Recht sind Teilbereiche zum Umgang mit der Leiche in verschiedenen Gesetzen geregelt.

Die Bundesverfassung entfaltet den Schutz der Leiche fragmentarisch im Lichte einzelner Bestimmungen. Zentral sind hierbei Art. 7 Menschenwürde, Art. 118 Schutz der Gesundheit, Art. 119a Transplantationsmedizin, Art. 122 Zivilrecht, Art. 123 Strafrecht, Art. 27 Wirtschaftsfreiheit, Art. 94 Grundsätze der Wirtschaftsordnung, Art. 95 Privatwirtschaftliche Erwerbstätigkeit.

Nach Art. 7 BV ist die Würde des Menschen zu achten und zu schützen. Diese Bestimmung wird ergänzt durch Art. 119a Abs. 1 BV, ${ }^{5}$ wonach die Menschenwürde auch im Bereich der Transplantationsmedizin zu schützen ist. Art. 118 b regelt die Forschung am Menschen, Art. 119a ermächtigt zudem den Bund zur Regelung der Transplantationsmedizin, und damit auch zum Umgang mit postmortal entnommenen Organen, Geweben und Zellen. Der Schutz der Bevölkerung vor Krankheitserregern, verursacht durch den Umgang mit Leichen, kann auf Art. 118 gestützt werden.

Aus den (zivil- und strafrechtlichen) Kompetenzen des Bundes zur Gesetzgebung auf dem Gebiet des Zivil- und Strafrechts, Art. 122 und Art. 123, lassen sich namentlich die folgenden Bereiche des Umgangs mit der Leiche einer Regelung zuführen: persönlichkeitsbezogene Aspekte beim Umgang mit der Leiche sowie der Einbezug von Angehörigen, z.B. bei der Entscheidung zu einer Organ- oder Gewebeentnahme; die Bestimmung des Todeszeitpunktes und die Zuständigkeit zur Todesfeststellung; die Unentgeltlichkeit der Organ- und Gewebespende als spezifische Beschränkung im Bereich des Vertragsrechts; allfällige Entnahmeverbote, datenschutzrechtliche Aspekte, Absicherung von Geboten und Verboten durch strafrechtliche Sanktionen. Die Grundsätze der Wirtschaftsfreiheit, Art. 27, eröffnen im Rahmen der Gesetze grds. auch den privatwirtschaftlichen Umgang mit Substanzen menschlicher Herkunft, Art. 94 f. ermöglichen dem Bund, gegen sozialschädliche Umtriebe Vorkehrungen zu entfalten. Diese Vorschriften können auch dem Schutz von Rechtsgütern wie Leben, Gesundheit, öffentlicher Ordnung und Sittlichkeit dienen.

5 Art. 119a Transplantationsmedizin lautet:

1_Der Bund erlässt Vorschriften auf dem Gebiet der Transplantation von Organen, Geweben und Zellen.

Er sorgt dabei für den Schutz der Menschenwürde, der Persönlichkeit und der Gesundheit.

2_Er legt insbesondere Kriterien für eine gerechte Zuteilung von Organen fest.

3_Die Spende von menschlichen Organen, Geweben und Zellen ist unentgeltlich. Der Handel mit menschlichen Organen ist verboten. 
Auf der Grundlage von Art. 119a BV wurde zum 1. Juli 2007 das Transplantationsgesetz $^{6}$ mit seinen Ausführungsverordnungen ${ }^{7}$ in Kraft gesetzt. Es dient u.a. der Umsetzung der bereits genannten EU-Richtlinien und vereinheitlichte die ehemals uneinheitlichen rechtlichen Voraussetzungen für die Spende, Entnahme und Übertragung von Organen, Geweben und Zellen auf eidgenössischer Stufe. Ziel des Gesetzes ist es, den missbräuchlichen Umgang der Transplantationsmedizin beim Menschen, insbesondere den Handel mit den Körpersubstanzen, zu verhindern und die Menschenwürde, die Persönlichkeit und die Gesundheit zu schützen, Art. 1 Abs. 3. Die wesentlichen Grundsätze der postmortalen Spende sind in Art. 8 ff. Transplantationsgesetz geregelt. Es gilt die erweiterte Zustimmungslösung. ${ }^{8}$ Sind keine nächsten Angehörigen vorhanden oder erreichbar, so ist die Entnahme unzulässig. ${ }^{9}$ Mindestalter der Spendenerklärung ist das sechzehnte Lebensjahr, Art. 8 Abs. 7.

Die postmortale Entnahme setzt den irreversiblen Ausfall aller Funktionen des Hirns und des Hirnstamms der spendenden Person voraus, sog. Gesamthirntod, Art. 9 Abs. 1. Die Festlegung der Hirntodkriterien ist dem Bundesrat zugewiesen, Art. 9 Abs. 2. Art. 7 der Transplantationsverordnung verweist weiter auf die medizinisch-ethischen Richtlinien der SAMW zur Feststellung des Todes mit Bezug auf Organtransplantationen in der Fassung vom 24. Mai 2005. Das Transplantationsgesetz ist auf europäischer Ebene eingebettet in das Biomedizinübereinkommen des Europarates und das Zusatzprotokoll bezüglich der Transplantation von Organen und Geweben menschlichen Ursprungs. Der Umgang mit der Leiche hat weiterhin Berührungspunkte zur Heilmittelgesetzgebung sowie zum Datenschutzrecht des Bundes und der Kantone.

Das Bundesgesetz über die Forschung am Menschen, das am 1. Januar 2014 in Kraft getreten ist, löste kantonale Regelungen ab und regelt die Forschung zu Krankheiten des Menschen sowie zu Aufbau und Funktion des menschlichen Körpers, durchgeführt an verstorbenen Personen, abschliessend. Vom Geltungsbereich werden ausschliesslich Forschungsprojekte erfasst. Die Verwendung von Leichen zu Aus-, Fort- und Weiterbildungszwecken sowie die Obduktion, soweit sie nicht auf die Forschung im oben beschriebenen Sinne abzielt, sind nicht erfasst.

6 Bundesgesetz vom 8. Oktober 2004 über die Transplantation von Organen, Geweben und Zellen (Transplantationsgesetz), SR 810.21.

7 Transplantationsverordnung, SR 810.211; Organzuteilungsverordnung, SR 810.212.4; Xenotransplantationsverordnung, SR 810.213; Transplantationsgebührenverordnung, SR 810.215.7; EDI-ZuteilungsVO, SR 810.212.41.

8 D.h. die postmortale Entnahme der Körpersubstanzen ist an die Bedingung geknüpft, dass die spendende Person vor ihrem Tod der Entnahme zugestimmt hat, Art. 8 Abs. 1. Liegt keine Willensäusserung vor, so geht das Bestimmungsrecht auf die nächsten Angehörigen über. Sie können unter Beachtung des mutmasslichen Willens der verstorbenen Person über das "ob" und "den Umfang" der Organentnahme entscheiden.

9 Der Kreis der ersatzweise zu befragenden «nächsten Angehörigen» bestimmt sich nach Art. 8 Abs. 8 Transplantationsgesetz sowie Art. 3 Transplantationsverordnung. 
Das Strafgesetzbuch regelt einige Straftatbestände, die festlegen, wann ein spezieller Umgang mit der Leiche bzw. mit dem Andenken an den Verstorbenen unzulässig ist. Der Schutzumfang ist jedoch fragmentarisch. Zu erwähnen sind die Straftatbestände Art. 262 StGB, Störung des Totenfriedens, und Art. 175 StGB, Üble Nachrede oder Verleumdung gegen einen Verstorbenen oder einen verschollen Erklärten. Gemäss Art. 262 StGB ist u.a. strafbar, wer einen Leichnam verunehrt oder öffentlich beschimpft oder wer einen Leichnam oder Teile eines Leichnams gegen den Willen des Berechtigten wegnimmt. Zusätzlich entfalten im Einzelfall noch weitere Tatbestände Relevanz: So z.B. die Eigentums- und Vermögensdelikte, Art. 137, 139 StGB. Die anhaltenden Diskussionen um die ggf. (un-) zulässige (Weiter-)Verwendung von Leichen- bzw. Fetalgewebe und jene über die Zulässigkeit und Grenzen von Sektionen werden von diesen Straftatbeständen nur unzureichend beantwortet. Denn sowohl die Sachqualität wie die Frage, ob die Leiche ggf. eigentumsfähig oder herrenlos ist, ist umstritten. Die Frage, unter welchen Voraussetzungen Gewebe, das legal, z.B. im Rahmen einer rechtsmedizinischen Sektion, entnommen wurde, für Forschungs- oder sonstige Zwecke aufbewahrt und weiterverwendet werden darf, findet daher auch im Strafrecht keine befriedigende Antwort. Die ärztliche Schweigepflicht, Art. 321 StGB, und das Forschungsgeheimnis, Art. $321^{\text {bis }}$ StGB, gelten auch nach dem Todeseintritt des Patienten bzw. Probanden.

Das Zivilrecht hält überwiegend nur allgemeine Bestimmungen zum Umgang mit der Leiche bereit. Zu erwähnen sind z.B. Art. 27 und 28 ZGB, Schutz der Persönlichkeit oder Art. $641 \mathrm{ff}$. ZGB, allgemeine Bestimmungen zum Sachenrecht. Wie bereits erwähnt, ist es aufgrund ihres ehemaligen Menschseins umstritten, ob die Leiche als Sache zu qualifizieren ist und wenn ja, ob sie grundsätzlich eigentumsfähig ist. Unter Einbezug der vielfältigen Verwendung von Leichenteilen in der Grundlagenforschung und der angewandten Forschung, aber auch der Pharmazie und den Medizinprodukten wäre es wünschenswert, wenn die Rechtsnatur der Leiche und die Bedeutung ihrer «menschlichen Herkunft» gesetzlich geklärt würden.

Abgesehen von den bundesrechtlichen Sonderregelungen unterfällt der Umgang mit dem toten Körper etc. der Gesetzgebungskompetenz der Kantone. Mit der Regelung des Bestattungswesens befassen sich ausserordentlich vielfältige Erlasse von Kantonen und Gemeinden. Aber auch die Gesundheitsgesetze sowie Patientinnen- und Patientengesetze enthalten Sonderregelungen zum Umgang mit der Leiche und zur Zulässigkeit z.B. von klinischen Obduktionen. ${ }^{10}$ Darüber hinaus begrenzen kantonale Polizeigesetze den Umgang mit der Leiche, indem dadurch z.B. unmittelbar drohende oder eingetretene schwere Störungen der öffentlichen Sicherheit und Ordnung abgewehrt oder beseitigt werden können.

10 Eine Zusammenstellung findet sich bei Bär W, Keller-Sutter T, Leichenschau, Obduktion und Transplantation, in: Poledna MW, Kuhn T (Hrsg.), Arztrecht in der Praxis, 2. Aufl. 2007, S. 767, 775 f. 
Wichtige Hilfestellung zum Umgang mit der Leiche bieten derzeit die untergesetzlichen Regelungsinstrumente, die überwiegend von medizinischen Fachgesellschaften verabschiedet wurden. Hier sind u.a. die Richtlinien und Empfehlungen der FMH und der SAMW zu nennen. Diese Regelwerke sind zwar kein Ersatz für Gesetze im formellen Sinne, zeigen aber notwendige Mindeststandards des Expertenwissens auf. Sie leisten damit einen wichtigen Beitrag bei der Auslegung der vorhandenen gesetzlichen Regelungen und geben Hinweise für mögliche künftige Neuregelungen. Exemplarisch ist hier auf die derzeit ausser Kraft gesetzten Richtlinien und Empfehlungen Biobanken: Gewinnung, Aufbewahrung und Nutzung von menschlichem biologischem Material für Ausbildung und Forschung und die Deklaration von Helsinki zu verweisen. Letztere hat aus sich heraus zwar keine unmittelbare Rechtswirkung, wurde aber durch das innerstaatliche Berufsrecht der Ärzte für anwendbar erklärt. ${ }^{11}$ Danach schliesst medizinische Forschung am Menschen die Forschung an identifizierbarem menschlichem Material oder identifizierbaren Daten ein. Die medizinische Forschung unterliegt ethischen Standards, welche die Achtung vor den Menschen fördern und ihre Gesundheit und Rechte schützen sollen, und darf nur von wissenschaftlich qualifizierten Personen und unter Aufsicht einer klinisch kompetenten, medizinisch ausgebildeten Person durchgeführt werden.

11 Art. 18 Standesordnung FMH i.V.m. Anhang 1 zur Standesordnung FMH. 
